International Journal of Trend in Scientific Research and Development (IJTSRD)

Volume: 3 | Issue: 2 | Jan-Feb 2019 Available Online: www.ijtsrd.com e-ISSN: 2456 - 6470

\title{
Smart Water Monitoring System using Cloud Service
}

\author{
Divya YA \\ Assistant Professor, Department of Information Science and Engineering, \\ GSSS Institute of Engineering and Technology for Women, Mysuru, India
}

\begin{abstract}
Water is the basic need for survival. Hence, the wastage of it is not tolerable. Water scarcity is the lack of sufficient available water resources to meet water needs within a region. Its effects are spread all over the world and around 2.8 billion people are affected by it. More than 1.2 billion people lack access to clean drinking water. Therefore, water monitoring has become an important subject of matter.
\end{abstract}

The project Water Monitoring System for Smart Village using cloud service, as the name says it is all about monitoring of water right from small villages, townships to entire urban infrastructure. The project deals with the efficient monitoring of water using Internet of Things (IoT) technology enabled by sensors. The sensor network can be flexible expanded and shrunk according to the requirements of setup. It is used for remotely controlling the water flow, cutting the water supply, monitoring and analyzing the water usage across the nodes, with the help of cloud connectivity. Further, more statistical data can be gathered and can be used by govt. authorities for defining policies, strategies and billing calculations. So ultimately, this will help to conserve and efficiently utilize the natural resource. Using IoT takes into account of waste wastage right from small village to large scale. It can also control the water usage in a precise way.

KEYWORDS: cloud, iot, monitor, water

\section{INTRODUCTION} INTERNET OF THINGS (IOT):

The Internet of Things (IoT) is a system of interrelated computing devices, mechanical and digital machines, objects, animals or people that are provided with unique identifiers and the ability to transfer data over a network without requiring human-to-human or human-to-computer interaction. Internet, a revolutionary invention, is always transforming into some new kind of hardware and software making it unavoidable for anyone. The form of communication that we see now is either human-human or human-device, but the Internet of Things (IoT) promises a great future for the internet where the type of communication is machine-machine (M2M).

The basic idea of IoT is to allow autonomous exchange of useful information between invisibly embedded different uniquely identifiable real world devices around us, fueled by the leading technologies like Radio-Frequency Identification (RFID) and Wireless Sensor Networks (WSNs) [2] which are sensed by the sensor devices and further processed for decision making, on the basis of which an automated action is performed [1]. IoT projects are under way that promise to improve distribution of the world's resources to those who need them most and help us understand our planet so we can be more proactive and less reactive.

\section{Methodology}

A. Architecture

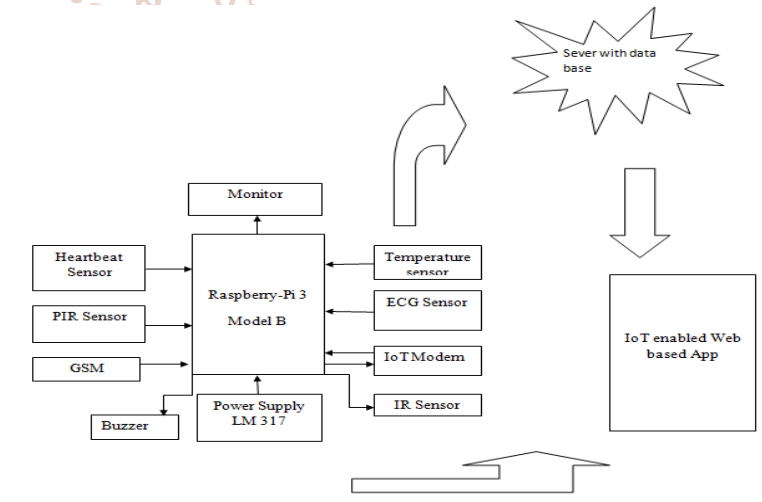

Fig 1: Block Diagram

Body Sensor Network (BSN) is the integration of intelligent, miniaturized low-power sensor nodes in, on or around human body to monitor body functions and the surrounding environment. Temperature sensor,Heart Beat sensor, ECG sensor,IR sensor are connected to Raspberry Pi through IC MCP3008. Heartbeat sensors arebased on the principle of photo phlethysmography. It measures the change in volume of blood through any organ of the body, which causes a change in light intensity through that organ. PIR sensor is a motion detection sensor, it detects whether the person is in movement or rest condition. IR sensor is used as obstacle sensor, which transmits an infrared signal, this infrared signal bounces from the surface of an object and the signal is received at the infrared receiver.

\section{B. Modules}

a. RASPBERRY PI3 B

Raspberry Pi 3provides the same Pi features but with double the ram and $6 \mathrm{x}$ faster processor speed. It is credit-card sizedcomputer capable of performing the multitask at single 
time. It runs on several flavors of Linux based operating system.

It consists of Broadcom BCM2836, an ARMv11 Quad Core ProcessorSystem-on-Chip, running at $1 \mathrm{Ghz}$, and a Videocore 4 GPU. The GPU provides Open GL ES 2.0, hardwareacceleratedOpenVG, and 1080p30 H.264 high-profile decode and is capable of $1 \mathrm{Gpixel} / \mathrm{s}, 1.5 \mathrm{Gtexel} / \mathrm{s}$ or 24 GFLOPs of generalpurpose compute. The biggestchange that has been enacted with the Raspberry Pi 3 isan upgrade to the main processor and an increase of ram from $512 \mathrm{MB}$ to $1 \mathrm{~GB}$.

\section{b. Temperature Sensor}

Temperature sensor is a gadget which detects varieties in temperature crosswise over it. LM35 is a fundamental temperature sensor that can be utilized for trial reason. It gives the readings in centigrade (degree Celsius). The LM35 series are exactness coordinated circuit temperature sensors, whose output voltage is proportional to the Celsius (Centigrade) temperature. It operates from 4 to 30 volts and has less than 60 Micro amperes current deplete.

\section{c. ECG Sensor}

ECG sensor is the pressure exerted by circulating blood upon the walls of blood vessels. When used without further specification, "blood pressure" usually refers to the arterial pressure in the systemic circulation. Blood pressure is usually expressed in terms of the systolic (maximum) pressure over diastolic (minimum) pressure and is measured in millimeters of mercury (mm Hg). Normal resting systolic (diastolic) blood pressure in an adult is approximately $120 \mathrm{~mm} \mathrm{Hg}(80 \mathrm{~mm} \mathrm{Hg})$, abbreviated "120/80 mm Hg". Blood pressure varies depending on situation, activity, and disease states. It is regulated by the nervous and endocrine systems. Blood pressure that is low due to a disease state is called hypotension, and pressure that is consistently high is hypertension.

\section{d. Heartbeat Sensor}

Heart rate is the speed of the heartbeat measured by the number of contractions of the heart per minute (bpm). The heart rate can vary according to the body's physical needs, including the need to absorb oxygen and excrete carbon dioxide. It is usually equal or close to the pulse measured at any peripheral point. Activities that can provoke change include physical exercise, anxiety, sleep, stress, illness, and ingestion of drugs. Many texts cite the normal resting adult human heart rate range from 60 to $100 \mathrm{bpm}$. Tachycardia is a fast heart rate, defined as above $100 \mathrm{bpm}$ at rest. Bradycardia is a slow heart rate, defined as below $60 \mathrm{bpm}$ at rest. The normal resting adult heart rate is probably closer to a range between 50 to $90 \mathrm{bpm}$. During sleep a slow heartbeat with rates around 40 to $50 \mathrm{bpm}$ is common and is considered normal. When the heart is not beating in a regular pattern, this is referred to as an arrhythmia. Abnormalities of heart rate sometimes indicate disease.

\section{e. PIR Sensor}

PIR sensors allows to sense motion, used to detect whether a human has moved in or out of the sensors range. They are small, inexpensive, low-power, easy to use and don't wear out. For that reason they are commonly found in appliances and gadgets used in homes or businesses. The PIR (Passive Infra-Red) Sensor is a pyroelectric device that detects motion by measuring changes in the infrared levels emitted by surrounding objects. This motion can be detected by checking for a high signal on a single I/O pin A PIR detector combined with a Fresnel lens are mounted on a compact size PCB together with an analog IC,SB0081, and limited components to form the module. High level output of variable width is provided. The PIR sensor has a detection range, ranging from 2-3meters. Supply voltage of 3$5 \mathrm{v}$.Current drain is less than $50 \mathrm{uA}$ Temperature ranges from $-15 \mathrm{C}$ to $+70 \mathrm{C}$.

\section{f. GSM [Global System for Mobile Communication]}

GSM module is used to establish communication between a computer and a GSM system. Global System for Mobile communication (GSM) is an architecture used for mobile communication for the users. GSM t enables higher data transmission rate for sending the alert messages when abnormalities occurs.

\section{g. Buzzer}

A buzzer or beeper is an audio signaling device, which may be mechanical, electromechanical, or piezoelectric. It is used for alerting when abnormalities occur to the patients.

\section{Workflow}

The Body sensor network architecture composed of wearable and implantable sensors. Each sensor node integrated with biosensors.These sensors collect the physiological parameters and forward them to a coordinator called Local Processing Unit (LPU), when the LPU detects any abnormalities then it provides immediate alert to the person. When the server receives data of a person from LPU, then it feeds the BSN data into its database and analyses those data. Subsequently, based on the degree of abnormalities, it may interact with the family members of the person, local physician, or emergency unit.

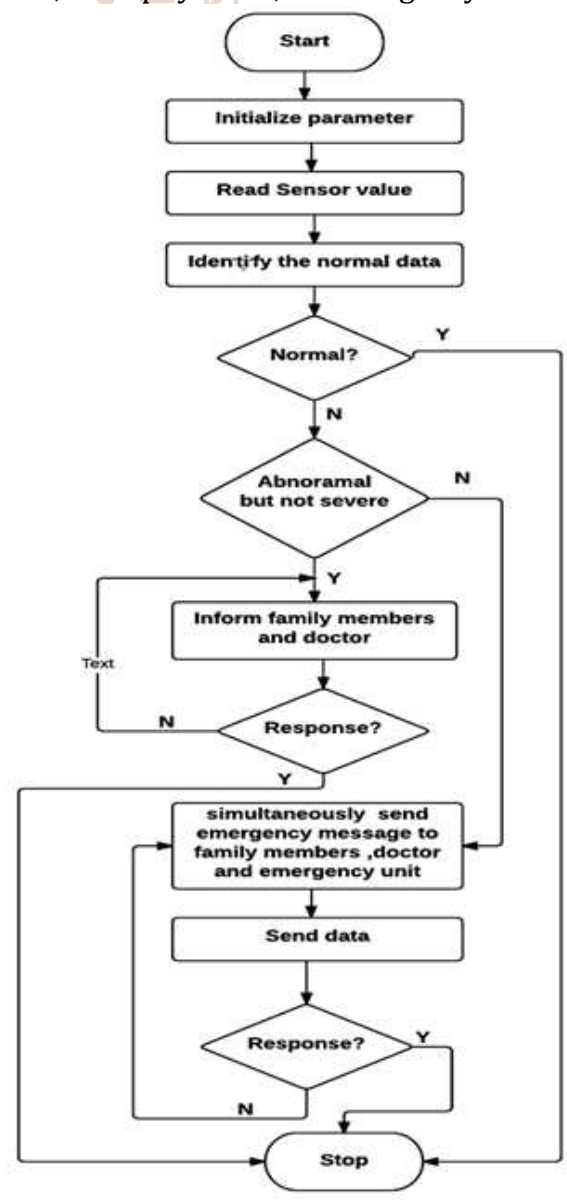

Fig 2: Workflow 


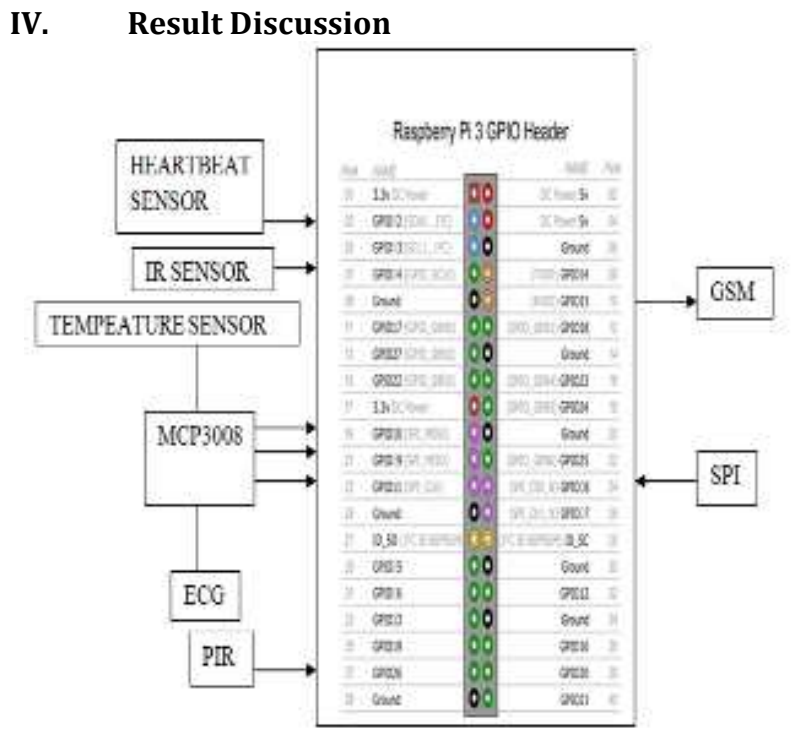

Fig3: Pin Configuration of Raspberry Pi 3 with sensors

Fig 3 shows Raspberry Pi 3 pins configured with sensors respectively. Heartbeat sensor is connected to GPIO 2, pin 3 where SDAI is function. IR sensor is connected to GPIO 4, pin 7, where GCLK is the function; PIR sensor is connected to pin 37 , GPIO 26; GSM is connected to pin 10 , GPIO 15 , where RXD0(UART) is its function. Temperature sensor and ECG sensor are connected to MCP3008 which is interfaced to SPI in Raspberry Pi 3 model B with pins 19,21,23, these pins are SPI pins functions as MOSI(SPI) for pin 19, MISO(SPI) for pin 21 and SCLK(SPI) for pin 23.SPI is Serial Peripheral Interface between IC MCP3008 and ADC respectively, the interface has to be done since analog values obtained must be converted to digital values.

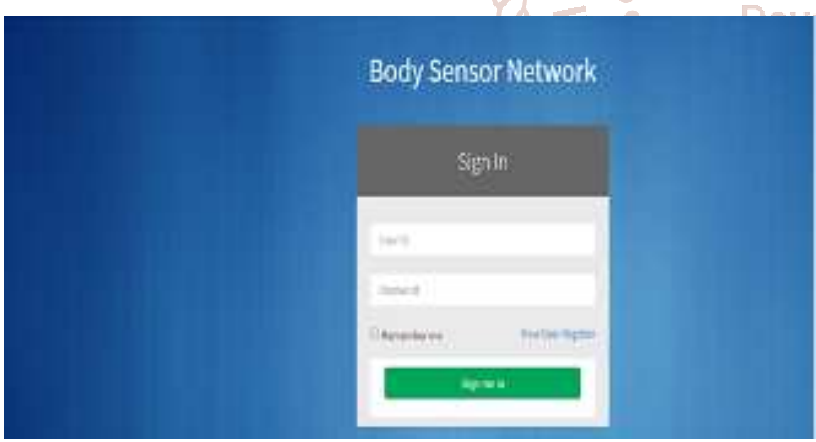

Fig 4: Sign in Page for BSN

Fig 4 shows sign-in using login ID and password in Body Sensor Network Web-app through internet in mobile or system which is convenient to the person respectively.

BOOY SENBOE METWOEKK

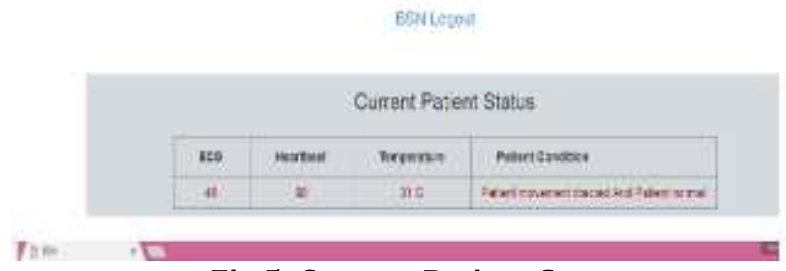

Fig 5: Current Patient Status

Fig 5 shows the current patient status were it contains the information of the different sensors values.

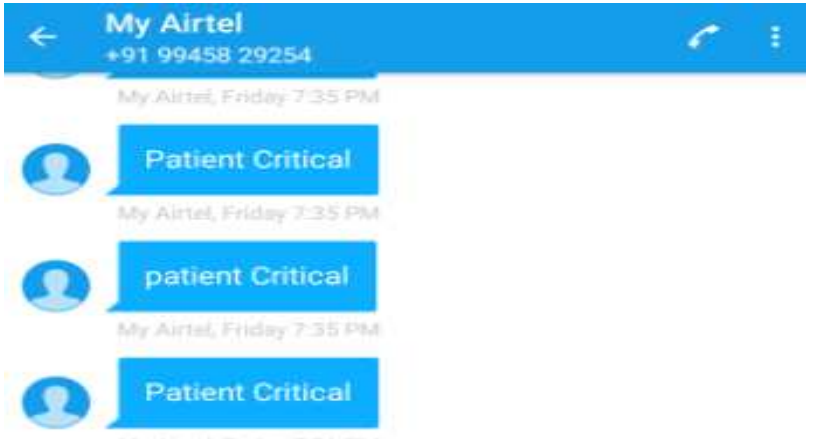

Fig6: Alert Message

Fig 6 shows the alert message to the recipient when the abnormal conditions occurs.

\section{CONCLUSION}

Health care services plays an important role in the society, automating these services lessen the burden on humans and eases the measuring process andalso the transparency of this system helps patients to trust it. When threshold value is reached, the alarm system that consists of buzzer, alerts the doctors, relatives and emergency unit based on the severity. The goal of developing monitoring framework is to reduce health care costs by reducing physician office visits, hospitalizations, and diagnostic testing procedure.

The GSM technology helps the server to update the patient data on website. Many further improvements can be made in the system to make it better and easily adaptable such as adding more advanced sensors and by developing the android application, which helps the user to use the application more conveniently.

\section{REFERENCES}

[1] World Population Ageing 2013, United Nations, New York, NY, USA, 2013, pp. 8-10.

[2] P. Gope and T. Hwang, "BSN-Care :A secure IoT-Based Modern Healthcare System Using Body Sensor Network," IEEE Sensors J., vol. 16, no. 5, Mar. 2016

[3] P. Kumar and H.-J. Lee, "Security issues in healthcare applications using wireless medical sensor networks: A survey," Sensors, vol. 12, no. 1, pp. 55-91, 2012.

[4] S M Riazhul Islam,Daehan Kwak, "The Internet of Things for Health Care: A Comprehensive Survey, "June 4, 2015

[5] K. Lorincz et al., "Sensor networks for emergency response: Challenges and opportunities," IEEE Pervasive Comput., vol. 3, no. 4, pp. 16-23, Oct./Dec. 2004.

[6] Himadri Nath Saha, Supratim Auddy, Subrata Pal," Health Monitoring using Internet of Things (IoT)",978-15386-2215-5/17,2017.

[7] Chris Otto, AleksandarMilenković, Corey Sanders, Emil Jovanov, "System Architecture Of A Wireless Body Area Sensor Network For Ubiquitous Health Monitoring", University of Alabama in Huntsville, Journal of Mobile Multimedia, Vol. 1, No.4 (2006) 307-326 (c) Rinton Press.

[8] Manoj Kumar, "An Analysis Of Authentication Schemes For Internet Of Things",An Analysis Of Authentication Schemes For Internet Of Things,ISSN: 22779655,[Kumar*, 4.(6): June, 2015.

[9] https://www.pythonforbeginners.com/ 